\title{
葡聚糖分子对氢氧化铁矿化结晶的调制作用
}

\author{
孙振亚 ${ }^{1,2 *} \quad$ 黄江波 $^{1}$ \\ ( ${ }^{1}$ 武汉理工大学资源与环境工程学院, 武汉 $430070 ;{ }^{2}$ 武汉理工大学材料研究与测试中心, 武汉 430070)
}

\begin{abstract}
摘要 通过对比五种不同葡聚糖浓度的 $\mathrm{Fe}^{3+}+$ 葡聚糖矿化作用体系中 $\mathrm{Fe}(\mathrm{OH})_{3}$ 凝胶在早期矿化阶段的成核和相 变过程来研究葡聚糖对铁矿物的结晶与转化的调控作用. 运用 ICP-AES(等离子发射光谱仪)观察各个矿化体系 中上清液的 $\left[\mathrm{Fe}^{3+}\right.$ 浓度及相应 $\mathrm{pH}$ 的变化, 矿化产物运用 FTIR、XRD 进行表征. 结果发现, 上清液中 $\left[\mathrm{Fe}^{3+}\right]$ 经历了 两次下降过程, 在陈化的第三天突然回增. 与不含葡聚糖的矿化作用体系很快形成结晶良好的 $\alpha$ - $\mathrm{FeOOH}$ 不同, 在含有葡聚糖的矿化作用体系中最初形成的物相主要为 $\beta$ - $\mathrm{FeOOH}$. 葡聚糖分子通过与 $\mathrm{Fe}^{3+}$ 配位吸附在铁氧化物 颗粒的表面促进了 $\beta$ - $\mathrm{FeOOH}$ 转化为 $\alpha-\mathrm{Fe}_{2} \mathrm{O}_{3}$. 相变是经过溶解-再结晶机制进行的. [ $\mathrm{Fe}^{3+}$ 第二次下降正是由于 $\beta-\mathrm{FeOOH}$ 转化为 $\alpha-\mathrm{Fe}_{2} \mathrm{O}_{3}$ 引起的. 葡聚糖浓度适当增大, $\beta-\mathrm{FeOOH}$ 溶解加快, 有利于相变的进行. 因此葡聚糖的 存在能显著影响在氢氧化铁凝胶中形成晶核的结构类型及其相变历程.
\end{abstract}

关键词: 生物矿化, 葡聚糖, $\mathrm{Fe}(\mathrm{OH})_{3}$ 凝胶, 相变, 溶解-再结晶

中图分类号： 0641

\section{Effect of Dextran on the Crystallization of Ferric Hydroxide}

\author{
SUN, Zhen-Ya ${ }^{1,2^{*}} \quad$ HUANG, Jiang-Bo ${ }^{1}$ \\ ('School of Resource and Environmental Engineering, Wuhan University of Technology, Wuhan 430070, P. R. China; \\ ${ }^{2}$ The Center of Research and Analysis of Materials, Wuhan University of Technology, Wuhan 430070, P. R. China)
}

\begin{abstract}
The initial nucleation and phase transformatin process of the $\mathrm{Fe}(\mathrm{OH})_{3}$ gel in five different mineralization systems with different dextran concentrations (from $0 \sim 2.0 \%(w)$ and the same $\left[\mathrm{Fe}^{3+}\right]=0.012 \mathrm{~mol} \cdot \mathrm{L}^{-1}$ ) have been investigated. The mineralizational phases in the initial stage were characterized by FTIR, XRD and ICP-AES spectrometry. The results showed that the concentration of $\mathrm{Fe}^{3+}$ ions in supernatant increased suddently in the third day during aging and then its secondary reduction stage occurred. There were the same chages of $\mathrm{pH}$ as $\left[\mathrm{Fe}^{3+}\right]$ in these process. The initial phases formed in the systems with dextran were mainly $\beta-\mathrm{FeOOH}$, not $\alpha$-FeOOH which growing rapidly in the system without dextran. The dextran molecules were adsorbed on the surfaces of iron oxide particles by complexing with $\mathrm{Fe}^{3+}$. The adsorbed dextran molecules would facilitate the phase transformation from $\beta$ - $\mathrm{FeOOH}$ into $\alpha-\mathrm{Fe}_{2} \mathrm{O}_{3}$ by a dissolution-recrystallization mechanism. The secondary reduction stage of $\left[\mathrm{Fe}^{3+}\right]$ corresponded to the process of the phase transformation from $\beta$ - $\mathrm{FeOOH}$ into $\alpha-\mathrm{Fe}_{2} \mathrm{O}_{3}$. With the increasement of the concentrations of dextran (up to $2.0 \%(w)$ ) in solution, the dissolution of $\beta$ - $\mathrm{FeOOH}$ and the formation of $\alpha-\mathrm{Fe}_{2} \mathrm{O}_{3}$ in these mineralization systems were accelerated. It was clear that dextran molecules could control the nuclei type and its phase transformation of the $\mathrm{Fe}(\mathrm{OH})_{3}$ gel.
\end{abstract}

Keywords: Biomineralization, Dextran, Ferric hydroxide gel, Transformation, Dissolution-recrystallization

生物矿化是一种普遍的生物现象, 从植物、微 生物直至动物体内, 均可形成矿物. 迄今为止, 在生
物体中已发现 60 余种不同的矿物, 由细菌产生的 矿物占总数的一半以上 ${ }^{[1]}$. 铁矿化物在表生环境中

Received: August 2, 2005; Revised:September 28, 2005. “Correspondent, E-mail:sunzy@ public.wh.hb.cn; Tel: 027-87651843.

国家自然科学基金(40372028, 40072015)资助项目

C. Editorial office of Acta Physico-Chimica Sinica 
广泛存在, 趋磁细菌中存在具有导向功能的磁铁矿, 嘉氏菌和纤发菌中有螺旋状和管状的针铁矿集合 体, 这都说明表生环境生物在氢氧化铁凝胶的形成 及其晶化过程中起着重要的作用. 生物细胞分泌的 有机基质主要为多糖和蛋白质, 研究表明在调制铁 的矿化过程中这些有机基质是极为重要的生物矿化 “活性剂”. 它们在有机-无机界面的分子识别, 在矿 物的成核、生长以及微结构的有序组装方面起着关 键作用.

近年来关于铁生物矿化的模拟实验已经进行了 不少, Kandori 等人利用 PEG、PVA、PAAm 和 PAAc 合成了不同形状的 $\alpha-\mathrm{Fe}_{2} \mathrm{O}_{3}$; Nesterova 等人 ${ }^{[3-4]}$ 利 用 $\operatorname{Alg}$ (藻肌酸) 自组装模板获得超顺磁性的纳米 $\mathrm{FeOOH}$; Sipos 等人 ${ }^{[5]}$ 利用壳聚糖为基底合成了球形 $\beta$ - $\mathrm{FeOOH}$.

氢氧化铁凝胶混合体系上清液中 $\left[\mathrm{Fe}^{3+}\right]$ 和 $\mathrm{pH}$ 值 的变化情况是研究氢氧化铁凝胶相变过程的两个重 要指标. 但关于这方面的研究还鲜见报道, 因此本实 验着重运用 ICP-AES 技术测定上清液的 $\left[\mathrm{Fe}^{3+}\right]$, 观察 其变化情况; 同时应用 FTIR、XRD 技术对矿化产物 进行物相分析. 通过对比有/无葡聚糖时两类不同矿 化作用体系中氢氧化铁凝胶的相变历程研究葡聚糖 分子对氢氧化铁凝胶早期矿化结晶行为的影响.

\section{1 实验部分}

\section{1 试剂与仪器}

无水 $\mathrm{FeCl}_{3}$ (中国医药集团上海化学试剂公司 ) 和氢氧化钠(天津市风船化学试剂科技公司)均为分 析纯, 葡聚糖(dextran, 中国医药集团上海化学试剂 公司)分子量 2 万; 实验用水为二次蒸馏水. 美国尼 高公司 NICOLET 60 SXB 型傅立叶变换红外光谱仪 (FTIR), 分辨率 $4 \mathrm{~cm}^{-1}$; 日本 RIGAKU 公司 D/MAX$R B$ 型 $X$ 射线衍射仪 $(X R D)$; 美国 Perkin Elmer 公司

\section{表 1 五种不同葡聚糖浓度(质量分数)的 $\mathbf{F e}^{3+} /$ 葡聚糖矿 化作用体系}

Table 1 Five kinds of $\mathrm{Fe}^{3+} /$ dextran mineralization systems with different concentrations of dextran at $(25 \pm 1){ }^{\circ} \mathrm{C}$

\begin{tabular}{cccc}
\hline Sample & $w($ dextran $)(\%)$ & {$\left[\mathrm{Fe}^{3+}\right] /\left(\mathrm{mol} \cdot \mathrm{L}^{-1}\right)$} & The initial $\mathrm{pH}$ \\
\hline A & 0 & 0.012 & 7.52 \\
B & 0.5 & 0.012 & 7.50 \\
C & 1.0 & 0.012 & 7.54 \\
D & 1.5 & 0.012 & 7.44 \\
E & 2.0 & 0.012 & 7.57 \\
\hline
\end{tabular}

Optima4300DV 型全谱直读等离子发射光谱仪(ICP$\mathrm{AES})$; 美国奥立龙公司 Orion818 型酸度计; 昆山市 超声波仪器有限公司 KQ-250B 型超声波清洗器. LG10-2.4A 型离心机(北京医用离心机厂).

\section{2 实验步骤}

分别将 $0.5 、 1.0 、 1.5 、 2.0 \mathrm{~g}$ 葡聚糖固体粉末溶于 $100 \mathrm{~mL}$ 二次蒸馏水中, 在 $60{ }^{\circ} \mathrm{C}$ 下超声震荡 $20 \mathrm{~min}$ 使其完全溶解, 冷却至室温, 再将 $8 \mathrm{~mL} \mathrm{FeCl}_{3}$ 溶液 $\left(0.3 \mathrm{~mol} \cdot \mathrm{L}^{-1}\right)$ 迅速加人准备好的葡聚糖溶液中, 超声 震荡 $10 \mathrm{~min}$ 使 $\mathrm{FeCl}_{3}$ 与葡聚糖混合均匀, 然后逐滴 加人 $\mathrm{NaOH}\left(0.1 \mathrm{~mol} \cdot \mathrm{L}^{-1}\right)$ 溶液, 调节 $\mathrm{pH}$ 至 7 , 混合溶 液搅拌均匀, 反应体系总体积控制在 $200 \mathrm{~mL}$. 此时 有凝胶状 $\mathrm{Fe}(\mathrm{OH})_{3}$ 形成. 待溶液稳定后, 在 $25{ }^{\circ} \mathrm{C}$ 恒温 下静置陈化. 溶液静置 $24 \mathrm{~h}$ 后, 上层清液与下层凝 胶严格分离. 定量吸取溶液下层凝胶液 $10 \mathrm{~mL}$, 每隔 一日取样一次, 连续取样七天. 样品经离心分离 $\left(8000 \mathrm{r} \cdot \mathrm{min}^{-1}\right)$ 后, 运用 ICP-AES 测定上清液 $\left[\mathrm{Fe}^{3+}\right]$ 浓 度, 同时测定其 $\mathrm{pH}$. 所得沉淀分别用二次蒸馏水洗 涤 2 次, 无水乙醇洗涤 1 次, 在 $60{ }^{\circ} \mathrm{C}$ 恒温下干燥 48 $\mathrm{h}$, 样品研碎后进行 FTIR 和 XRD 物相分析. 结果见 表 1 .

\section{2 结果与讨论}

\section{1 不同反应体系氢氧化铁凝胶的相变历程}

氢氧化铁凝胶在有/无葡聚糖存在的两类不同 矿化作用体系中陈化, 陈化开始后的前七天混合溶 液上清液的 $\left[\mathrm{Fe}^{3+}\right]$ 和 $\mathrm{pH}$ 变化情况如图 1、图 2 所示.

在不含葡聚糖的矿化作用体系中 (见图 la), 氢

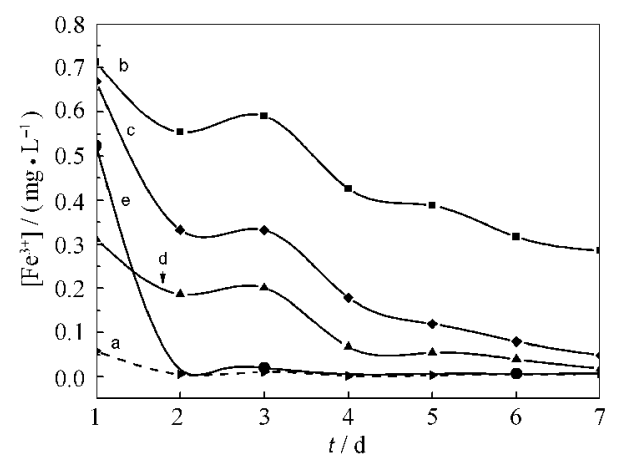

图 1 不同矿化作用体系中上清液的 $\left[\mathrm{Fe}^{3+}\right]$ 随陈化时间 的变化

Fig.1 Changes of the concentrations of $\mathrm{Fe}^{3+}$ in the supernatant of different systems with the aging days

(a) sample A, (b) sample B, (c) sample C, (d) sample D, (e) sample E 


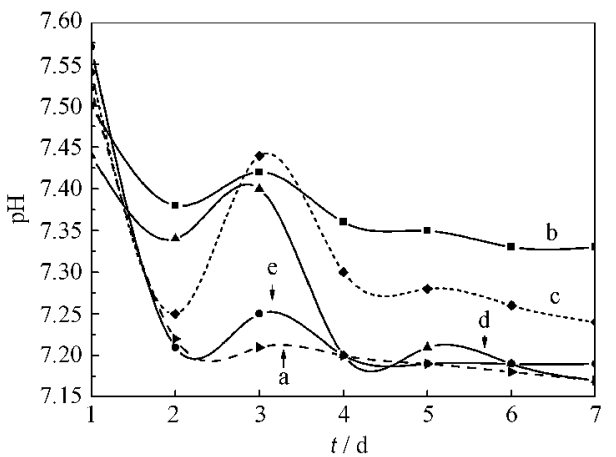

图 2 不同矿化作用体系中上清液的 pH 随陈化时间 的变化

Fig.2 Changes of the $\mathrm{pH}$ in the supernatant of different systems with the aging times

(a) sample A, (b) sample B, (c) sample C, (d) sample D, (e) sample E

氧化铁沉淀反应后上清液初始 $\left[\mathrm{Fe}^{3+}\right]$ 即降至很低, 陈 化开始后的前两天, $\left[\mathrm{Fe}^{3+}\right]$ 迅速降低, 在第三天 $\left[\mathrm{Fe}^{3+}\right]$ 略有回升, 对应溶液的 $\mathrm{pH}$ 值显示了相同的情况(见图 $2 \mathrm{a})$. 刚开始 $\left[\mathrm{Fe}^{3+}\right]$ 和 $\mathrm{pH}$ 值迅速降低是由于 $\mathrm{Fe}(\mathrm{OH})_{3}$ 转化为 $\alpha-\mathrm{FeOOH}$ 引起的. 图 3 为样品 $\mathrm{A}$ 分别陈化 了 $3 \mathrm{~d}$ 和 $20 \mathrm{~d}$ 的红外谱图. 由图 3 可见, 在陈化的第 三天已经开始出现弱的 $\alpha-\mathrm{FeOOH}$ 特征峰 $893 、 800$ $\mathrm{cm}^{-1}$, 陈化 $20 \mathrm{~d}$ 后, 上述两峰强度明显增大, 说明结 晶度进一步提高, 电镜观察表明长针状晶体逐渐长 大, 大小约为 $20 \mathrm{~nm}$ (图略), 但晶体结构并未改变. 因 此随着陈化时间的延续, $\alpha-\mathrm{FeOOH}$ 逐渐稳定, 吸附在 $\alpha$ - $\mathrm{FeOOH}$ 表面上的不稳定 $\mathrm{Fe}(\mathrm{III})$ 水合物如 $\mathrm{Fe}(\mathrm{OH})^{2+}$ 、 $\mathrm{Fe}(\mathrm{OH})_{2}^{+} 、 \mathrm{Fe}_{2}(\mathrm{OH})_{2}^{4+}$ 等逐步解吸释放到水相中, 使得 随后上清液中 $\left[\mathrm{Fe}^{3+}\right]$ 略有回升, 溶液 $\mathrm{pH}$ 值相应缓慢

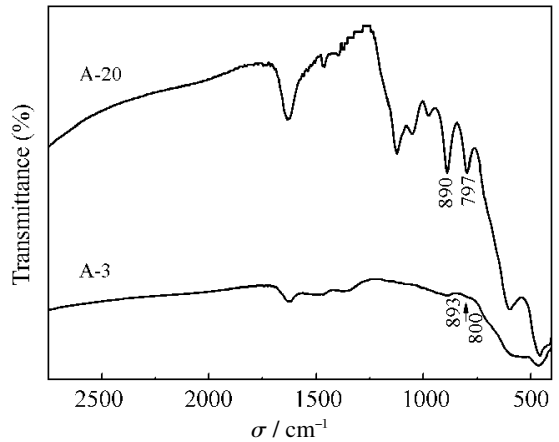

图 3 不同陈化时间样品 $\mathbf{A}$ 的红外图谱

Fig.3 FTIR spectra of sample A after different aging times

A-3: after aging 3 days, A-20: after aging 20 days
下降(见图 2a).

而含有葡聚糖的矿化作用体系在陈化开始后的 前 7 天, 上清液中的 $\left[\mathrm{Fe}^{3+}\right]$ 都经历了一个先快速下降, 再略微上升, 然后又第二次下降的过程(见图 lb le), 这可能与体系中铁(氧)氢氧化物发生相变有关. 第 一次 $\left[\mathrm{Fe}^{3+}\right]$ 快速下降发生在陈化开始后的第 $1 \sim 2 \mathrm{~d}$, 第 $3 \mathrm{~d}\left[\mathrm{Fe}^{3+}\right]$ 略有回升, 第二次下降过程在第 $3 \sim 7 \mathrm{~d}$, 在第 $7 \mathrm{~d}\left[\mathrm{Fe}^{3+}\right]$ 已经非常低, 对应溶液 $\mathrm{pH}$ 也显示了 相同的变化趋势(见图 $2 \mathrm{~b} \sim 2 \mathrm{e}$ ).

陈化开始时, 初始 $\left[\mathrm{Fe}^{3+}\right]$ 在含有葡聚糖的矿化作 用体系中要明显高于不含葡聚糖的矿化作用体系， 这说明吸附在氢氧化铁凝胶表面的葡聚糖增大了 $\mathrm{Fe}(\mathrm{OH})_{3}$ 的溶解度. 当葡聚糖浓度增大 (由 $\mathrm{b} \sim \mathrm{e}$ ), 初始 $\left[\mathrm{Fe}^{3+}\right]$ 有所降低, 这说明葡聚糖对铁(氧)氢氧化物有 包裹作用, 阻碍了 $\mathrm{Fe}^{3+}$ 向水相扩散. 但由图 1 可见, 反 应体系中葡聚糖浓度的改变并没有改变 $\left[\mathrm{Fe}^{3+}\right]$ 第二 次下降开始的时间, 都在第三天开始下降, 葡聚糖对 铁(氧)氢氧化物的包裹作用并未影响成核和相变的 进程.

我们选择对葡聚糖浓度较大的矿化作用体系不 同陈化时间的样品进行了红外光谱研究. 图 4 是 $\mathrm{E}$ 样品分别陈化了 $1 、 3$ 和 $20 \mathrm{~d}$ (分别记为 E- $1 、 E-3$ 和 E-20)后的红外谱图. 图中 $3421 \mathrm{~cm}^{-1}$ 归属于 $\mathrm{O}-\mathrm{H}$ 的伸缩振动, $1640 \mathrm{~cm}^{-1}$ 归属于水分子的吸收峰, $2922 、 2852 \mathrm{~cm}^{-1}$ 归属于 $\mathrm{C}-\mathrm{H}$ 的伸缩振动, $1365 \mathrm{~cm}^{-1}$ 归属于 $\mathrm{H}-\mathrm{C}-\mathrm{OH}$ 的弯曲振动, $1156 \mathrm{~cm}^{-1}$ 归属于 $\alpha$-糖苷桥 $(\mathrm{C}-\mathrm{O}-\mathrm{C})$ 的不对称伸缩振动, $910 、 845$ 、 $760 \mathrm{~cm}^{-1}$ 则对应的是 $\alpha$-吡喃型葡聚糖环变形模式.

由于 $\mathrm{Fe}(\mathrm{III})$-葡聚糖配合物的主要成分骨架均

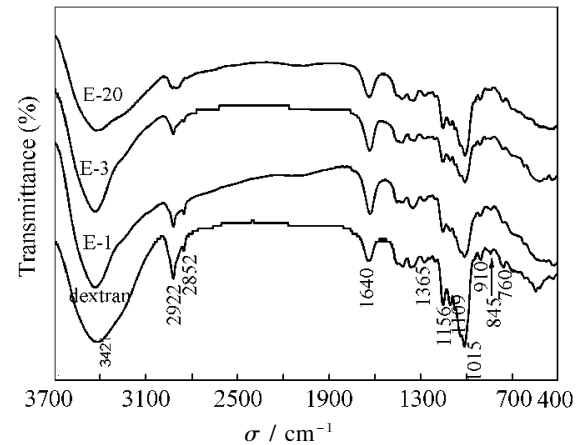

图 4 不同陈化时间样品 $\mathbf{E}$ 与纯葡聚糖样品的红外图谱 比较

Fig.4 Comparison of the FTIR spectra of sample E after different aging times and pure dextran E-1:after aging 1 day, E-3:after aging 3 days, E-20: after aging 20 days 


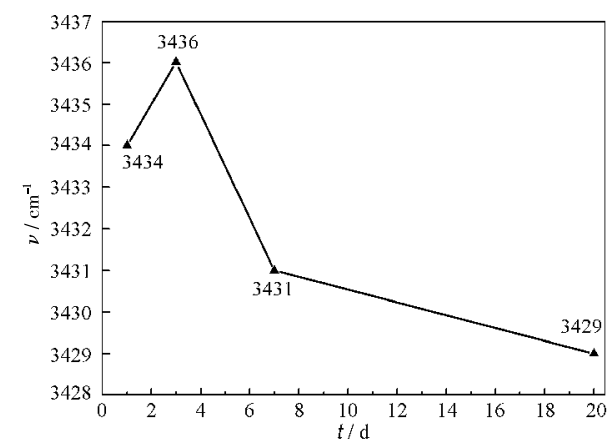

图 5 样品 $\mathbf{E}$ 的 $\nu_{\mathrm{OH}}$ 伸缩振动吸收峰随陈化时间变化

Fig.5 Changes of the wavenumber ascribed to $\mathbf{0}-\mathbf{H}$ stretching vibration in sample $\mathbf{E}$ with the aging times

为葡聚糖, 所以它与纯葡聚糖的红外谱图基本相似, 只有少数几个谱带发生了变化. 除在 500 1200 $\mathrm{cm}^{-1}$ 明显变宽外, 在 $3421 、 1365 \mathrm{~cm}^{-1}$ 还发生了较大的位 移. $1109 \mathrm{~cm}^{-1}$ 附近的吸收峰属于葡萄糖残基 C-4 位 置上的 $\mathrm{C}-\mathrm{O}$ 振动. 1040 和 $1015 \mathrm{~cm}^{-1}$ 分别与葡聚 糖存在的状态, 即晶态或者非晶态有关. $1040 \mathrm{~cm}^{-1}$ 逐渐减弱, 说明与纯葡聚糖相比, 吸附在纳米铁氧化 物颗粒表面上的葡聚糖是无序的非晶相 ${ }^{[6}$.

随着陈化时间的延长, 红外分析表明, 原来纯葡 聚糖位于 $3421 \mathrm{~cm}^{-1}$ 的 $\mathrm{O}-\mathrm{H}$ 的伸缩振动呈现向高 波数方向规律性地移动, 其变化情况如图 5 所示. 该 谱带向高频区位移的原因与葡聚糖大分子链上羟基 的氢原子脱去, 发生氧上的电子转移导致 $\mathrm{C}-\mathrm{O}$ 键 的键力常数变大有关, 表明配合物中配体葡聚糖的 部分羟基氧原子被 $\mathrm{Fe}$ (III) 取代形成 $\mathrm{C}-\mathrm{O}-\mathrm{Fe}$ 键. 其吸收峰强度随陈化时间逐渐减弱, 说明羟基数量 不断减少. 同时 $1365 \mathrm{~cm}^{-1}$ 仲羟基的弯曲振动吸收峰 均往低波数的方向位移, 这是因为葡聚糖中仲羟基 的氧原子与 $\mathrm{Fe}(\mathrm{III})$ 发生配位作用而使 $\mathrm{C}-\mathrm{O}$ 键削 弱. 此外, 在反映糖类构型的谱带 930 750 $\mathrm{cm}^{-1}$ 也 发生了较大变化, $910 \mathrm{~cm}^{-1}$ 向高波数位移至 $916 \mathrm{~cm}^{-1}$ 左右, $845 、 760 \mathrm{~cm}^{-1}$ 强度明显减弱, 说明 $\mathrm{Fe}(\mathrm{III})$ 的加 人改变了葡聚糖的结构, 葡聚糖的环状结构将展开, 有利于铁离子在上边成核、结晶. 因此葡聚糖与 $\mathrm{Fe}(\mathrm{III})$ 如 $\mathrm{Fe}^{3+} 、 \mathrm{Fe}(\mathrm{OH})^{2+} 、 \mathrm{Fe}(\mathrm{OH})_{2}^{+}$等之间存在配位相互作用, 作用位点主要在葡聚糖分子的羟基基团上 ${ }^{[7]}$.

图 4E-1 中 550 和 $478 \mathrm{~cm}^{-1}$ 可归属于 $\alpha-\mathrm{Fe}_{2} \mathrm{O}_{3}$ 的 $\mathrm{Fe}-\mathrm{O}$ 振动, 与标准 $\alpha-\mathrm{Fe}_{2} \mathrm{O}_{3}$ 晶体特征峰相比, 这 两个峰比较宽且弱, 说明 E-1 样品中含有非晶态的 铁氧化物颗粒, XRD 分析证实其为非晶态物质 ${ }^{[8]}$ (见 图6a), 结构类似于许多文献报道过的水合氧化铁(2line ferrihydrite). 陈化三天后样品 E-3 中 546 和 482 $\mathrm{cm}^{-1}$ 比 E-1 明显增强, 此外 E-3 中还出现了 $874 \mathrm{~cm}^{-1}$ 和 $700 \mathrm{~cm}^{-1}$ 两个弱的 $\beta-\mathrm{FeOOH}$ 特征吸收峰, 说明 在 $\mathrm{Fe}(\mathrm{OH})_{3}$ 凝胶中开始出现铁氧氢氧化物晶核. 随 着陈化时间的延长, 在 E-20 中 $874 \mathrm{~cm}^{-1}$ 减弱, 而 $700 \mathrm{~cm}^{-1}$ 几乎消失, 说明样品中 $\beta-\mathrm{FeOOH}$ 的含量减 少, 同时新出现 $593 \mathrm{~cm}^{-1}$, 可归属于 $\alpha-\mathrm{Fe}_{2} \mathrm{O}_{3}$ 的特征 峰. XRD 进一步证实样品中除 $\beta-\mathrm{FeOOH}$ 外还存在 $\alpha-\mathrm{Fe}_{2} \mathrm{O}_{3}$ 晶相(见图 $6 \mathrm{~b}$ ), 表明在 $\mathrm{Fe}(\mathrm{OH})_{3}$ 凝胶的早期 矿化阶段发生了相变.

因此在含有葡聚糖的矿化作用体系中, 刚开始 $\left[\mathrm{Fe}^{3+}\right]$ 急剧下降是因为 $\beta-\mathrm{FeOOH}$ 大量成核. 随后 $\beta$ $\mathrm{FeOOH}$ 的溶解使得 $\left[\mathrm{Fe}^{3+}\right]$ 略有上升. 葡聚糖浓度增 大, $\left[\mathrm{Fe}^{3+}\right]$ 上升的比例相应增大, 葡聚糖浓度由样品 $\mathrm{B}$ 的 $0.5 \%(w)$ 上升到样品 $\mathrm{E}$ 的 $2.0 \%(w),\left[\mathrm{Fe}^{3+}\right]$ 上升的比 例相应由样品 $\mathrm{B}$ 的 $6.7 \%$ 上升到样品 $\mathrm{E}$ 的 $17.6 \%$, 说 明葡聚糖浓度增大加快了 $\beta-\mathrm{FeOOH}$ 溶解的速率. $\beta$ $\mathrm{FeOOH}$ 的溶解, 上清液中的 $\left[\mathrm{Fe}^{3+}\right]$ 理应上升, 但 ICPAES 测试的结果表明, 在含有葡聚糖的体系中, $\left[\mathrm{Fe}^{3+}\right]$ 在 $\beta-\mathrm{FeOOH}$ 溶解后却出现了第二次下降过程, 这是由于 $\beta-\mathrm{FeOOH}$ 迅速转化成了 $\alpha-\mathrm{Fe}_{2} \mathrm{O}_{3}{ }^{[0-10]}$. $\left[\mathrm{Fe}^{3+}\right]$ 的这种变化说明在含有葡聚糖的体系中相变是经 过溶解-再结晶机制进行的.

\section{2 葡聚糖分子作用下 $\mathbf{F e}(\mathrm{OH})_{3}$ 矿化结晶机理讨论}

葡聚糖是亲水性的多羟基聚合物. 在形成氢氧 化铁凝胶的同时, 葡聚糖分子通过与铁氧化物颗粒 表面形成氢键的方式吸附在其表面. 葡聚糖中的羟 基与 $\mathrm{Fe}(\mathrm{III})$ 发生配位相互作用形成 $\mathrm{Fe}(\mathrm{III})$ - 葡聚糖 配合物。

与不含葡聚糖的矿化作用体系很快形成结晶良

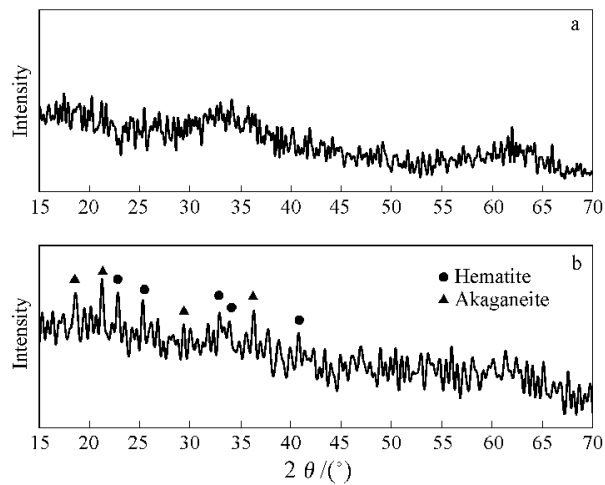

图 6 不同陈化时间的样品 $\mathbf{E}$ 的 XRD 谱图

Fig.6 XRD patterns of sample $E$ after different aging times: aging 1d (a), aging $20 \mathrm{~d}$ (b) 
好的 $\alpha-\mathrm{FeOOH}$ 不同, 葡聚糖的存在一方面调控了沉 淀物相的晶体结构, 使得初始时形成的物相主要为 $\beta$-FeOOH 而非 $\alpha-\mathrm{FeOOH}$. 在形成 $\beta$ - $\mathrm{FeOOH}$ 晶核之 前, 我们发现样品中含有非晶态的铁氧化物颗粒. 我 们的诸多实验表明, 当进一步加大葡聚糖浓度或使 用更高分子量的壳聚糖调控其矿化作用时, 其结晶 相主要为 $\beta-\mathrm{FeOOH}$, 但晶形和粒度则相应被改变. 另一方面葡聚糖的存在促进了 $\beta-\mathrm{FeOOH}$ 转化为 $\alpha-\mathrm{Fe}_{2} \mathrm{O}_{3}$.

$\beta$ - $\mathrm{FeOOH}$ 被认为是通过 $\mathrm{Fe}(\mathrm{OH})_{3}$ 的溶解-再结 晶过程形成的. 即 $\mathrm{Fe}(\mathrm{OH})_{3}$ 凝胶首先溶解成离子进 人溶液, 溶解产生的 $\mathrm{Fe}(\mathrm{III})$ 离子可以 $\mathrm{Fe}^{3+} 、 \mathrm{Fe}(\mathrm{OH})^{2+}$ 、

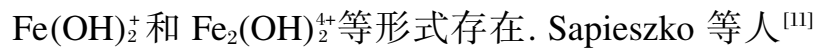
的研究表明, 在低温中性水溶液中 $\mathrm{Fe}$ (III) 离子主 要以 $\mathrm{Fe}(\mathrm{OH})_{2}^{+}$的形式存在. 这些从氢氧化铁凝胶中 脱附下来的 $\mathrm{Fe}(\mathrm{OH})_{2}^{+}$迅速水解、团聚生成铁的团聚 体, 称其为初级粒子, 这些初级粒子很不稳定, 在 $\mathrm{Fe}(\mathrm{OH})_{3}$ 的表面聚集达到一定数量后, 在粒子间范德 华力及磁性力作用下, 相邻粒子间相互吸引、团聚, 最终转化为 $\mathrm{FeOOH}$. 这是一个去质子的过程, 因此 $\mathrm{pH}$ 不断下降. 由于形成晶态的 $\mathrm{FeOOH}$ 相对于凝胶 状的 $\mathrm{Fe}(\mathrm{OH})_{3}$ 溶解度要小很多, 溶液中的 $\left[\mathrm{Fe}^{3+}\right]$ 同样 不断下降. 在含有葡聚糖的体系中, 由于葡聚糖分子 很容易吸附到铁氧化物的表面, 使得局部 $\mathrm{Cl}^{-}$浓度相 当高, 容易获得 $\beta$ - $\mathrm{FeOOH}$, 这与许多文献报道的结

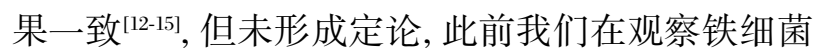
中的矿化物时发现主要晶相为 $\alpha-\mathrm{FeOOH}^{[16]}$, 这是否 与模拟生物矿化实验中存在 $\mathrm{Cl}^{-}$有关还有待进一步 的研究. 而在不含葡聚糖的体系中, 情况有所不同, 当溶液 $\mathrm{pH}$ 在 7 8 之间, $\mathrm{Cl}^{-}$大部分已从 $\mathrm{Fe}(\mathrm{III})$ 聚合 物中释放出来, 形成的往往是 $\alpha-\mathrm{FeOOH}^{[17]}$, 说明葡聚 糖分子能调控 $\mathrm{Fe}(\mathrm{III})$ 的结晶类型. 不同晶型 $\mathrm{FeOOH}$ 的 稳定性不同, 因此其溶解速率也不相同, 越稳定的 $\mathrm{FeOOH}$ 溶解越困难. $\alpha$ - $\mathrm{FeOOH}$ 是最稳定的铁氧氢 氧化物, 一旦形成就很难在低温下转化为 $\alpha-\mathrm{Fe}_{2} \mathrm{O}_{3}$.

Sugimoto 等人 ${ }^{198}$ 认为最有可能成为形成 $\alpha-\mathrm{Fe}_{2} \mathrm{O}_{3}$ 的前驱体是 $\mathrm{Fe}(\mathrm{OH})_{2}^{+}$, 但 $\mathrm{pH}$ 在 7 8 时的氢氧化铁 凝胶溶液中 $\mathrm{Fe}(\mathrm{OH})_{2}^{+}$十分稀少, 因此 $\alpha-\mathrm{Fe}_{2} \mathrm{O}_{3}$ 的成核 和生长很难从溶液中直接获得足够的溶质, 它需要 溶解 $\beta$ - $\mathrm{FeOOH}$ 为其提供溶质. 最初 $\beta$ - $\mathrm{FeOOH}$ 溶解 使得 $\left[\mathrm{Fe}^{3+}\right.$ 略有回升, 是因为纤维状 $\beta-\mathrm{FeOOH}$ 的存 在会阻碍初级粒子向 $\alpha-\mathrm{Fe}_{2} \mathrm{O}_{3}$ 的转移. 随着纤维状 $\beta-\mathrm{FeOOH}$ 逐渐溶解完毕, 相变加速进行, $\left[\mathrm{Fe}^{3+}\right]$ 继续
下降, 因而出现了第二次的下降过程.

葡聚糖浓度增大, $\beta$ - $\mathrm{FeOOH}$ 溶解加快, 有利于 相变的进行, 因此在一定范围内葡聚糖浓度增大有 利于晶体的生长, 可能是因为葡聚糖浓度增大, 对初 始晶核的包裹作用增强, 使得晶核的粒度较小而易 于溶解. 但葡聚糖浓度过大又会抑制成核, 这与我们 先前利用更高分子量的壳聚糖(CS)合成 $\beta-\mathrm{FeOOH}$ 的实验结果相似, 在一定范围内 $(\mathrm{CS}<0.6 \%(w))$ 壳聚 糖浓度增大有利于晶体的生长, 但壳聚糖浓度过大 (如升至 1.2\%(w)) 却又抑制晶体的成核和生长 ${ }^{[19]}$.

\section{3 结 论}

葡聚糖的存在能显著影响在氢氧化铁凝胶中形 成晶核的结构类型及其相变历程. 与不含葡聚糖的 矿化作用体系很快形成结晶良好的 $\alpha-\mathrm{FeOOH}$ 不同, 在含有葡聚糖的矿化作用体系中最初形成的物相主 要为 $\beta$ - $\mathrm{FeOOH}$. 葡聚糖含有大量的羧基、醇羟基、醚 基等铁离子潜在的结合键, 当铁离子与葡聚糖作用 时, 红外光谱分析表明铁离子主要与糖上羟基发生 配位作用. 葡聚糖分子吸附在铁氧化物颗粒的表面 促进了 $\beta-\mathrm{FeOOH}$ 转化为 $\alpha-\mathrm{Fe}_{2} \mathrm{O}_{3}$. 相变是经过溶解再结晶机制进行的, ICP-AES 测定体系上清液中 $\left[\mathrm{Fe}^{3+}\right]$ 出现第二次下降过程证实了这一点. 葡聚糖浓 度适当增大, $\beta-\mathrm{FeOOH}$ 溶解加快, 有利于相变的进 行. 事实上, 在海洋软体动物石鳖的齿尖上除磁铁 矿、针铁矿外还共存少量 $\alpha-\mathrm{Fe}_{2} \mathrm{O}_{3}{ }^{[20]}$, 本实验结果有 助于解释在自然界中生物多糖对铁矿物的结晶与转 化的调控作用.

\section{References}

1 Ouyang, J. M. Progress in Chemistry, 2005, 17: 749 [欧阳健明. 化学进展(Huaxue Jinzhan), 2005, 17 : 749]

2 Kandori, K.; Yamoto, Y.; Ishikawa, T. J. Colloid Interf. Sci., 2005, 283 : 432

3 Nesterova, M. V.; Walton, S. A.; Webb, J. J. Inorg. Biochem., 2000, 79:109

4 Nesterova, M. V.; Moreau, J.; Banfield, J. F. Geochim. Cosmochim. Acta, 2003, 67 : 1177

5 Sipos, P.; Berkesi, O.; Tombácz, E.; Pierre, T. G. S.; Webb, J. J. Inorg. Biochem., 2003, 95: 55

6 Xu, X. Q.; Shen, H.; Xu, J. R.; Xu, J.; Li, X. J.; Xiong, X. M. Appl. Surf. Sci., 2005, 5: 1

7 Bautista, M. C.; Bomati-Miguel, O.; Morales, M. P.; Serna, C. J.; 
Veintermillas-Verdaguer, S. J. Magn. Magn. Mater., 2005, 293 : 20

8 Music, S.; Santana, G. P.; Smit, G.; Garg, V. K. J. Alloys Compd., 1998, 278: 291

9 Sugimoto, T.; Sakata, K.; Muramatsu, A. J. Colloid Interf. Sci., 1993, 159: 372

10 Sugimoto, T.; Muramatsu, A. J. Colloid Interf. Sci., 1996, 184: 626

11 Sapieszko, R. S.; Patel, R. C.; Matijevic, E. J. Phys. Chem., 1977, 81: 1061

12 Mackay, A. L. Miner. Mag., 1960, 32 : 547

13 Ellis, J.; Giovanoli, R.; Stumm, W. Chimica., 1976, 30: 141

14 Cornell, R. M.; Giovanoli, R. Clays Clay Miner., 1988, 36: 385

15 Chambaere, D. G.; de Grave, E. Phys. Chem. Miner., 1985, 12 :
176

16 Che, Y.; Wang, L. Z.; Sun, Z. Y.; Chen, J. Z.; Du, G.; Wu, S. C. Geosci., 2002, 16: 305 [车 遥, 王利忠, 孙振亚, 陈敬中, 杜 刚, 吴书成. 现代地质(Xiandai Dizhi), 2002, 16: 305]

17 Liu, H.; Wei, Y.; Sun, Y. H.; Wei, W. Coll. Surf. A: Physicochem. Eng. Aspects, 2005, 252: 201

18 Sugimoto, T.; Sakata, K.; Muramatsu, T. J. Colloid Interf. Sci., 1993, 159:372

19 Chen, H. S.; Sun, Z. Y.; Liu, Q. L.; Zen, J. M. Centeral South Pharmacy, 2005, 3: 3 [陈和生, 孙振亚, 刘巧灵, 曾俊敏. 中南 药学(Zhongnan Yaoxue), 2005, 3: 3]

20 Liu, C. L.; Zhao, J. G.; Gong, B. A. Acta Biophys. Sin., 2002, 16: 655 [刘传琳, 赵见高, 宫宝安. 生物物理学报(Shengwu Wuli Хиеьao), 2002, 16: 655] 